\title{
Parallel Integral Projection Transform for Straight Electrode Localization in 3-D Ultrasound Images
}

\author{
Martin Barva, Marián Uherčík, Jean-Martial Mari, Jan Kybic, Member, IEEE, Jean-René Duhamel, \\ Hervé Liebgott, Václav Hlaváč, and Christian Cachard
}

\begin{abstract}
In surgical practice, small metallic instruments are frequently used to perform various tasks inside the human body. We address the problem of their accurate localization in the tissue. Recent experiments using medical ultrasound have shown that this modality is suitable for real-time visualization of anatomical structures as well as the position of surgical instruments. We propose an imageprocessing algorithm that permits automatic estimation of the position of a line-segment-shaped object. This method was applied to the localization of a thin metallic electrode in biological tissue. We show that the electrode axis can be found through maximizing the Parallel Integral Projection transform that is a form of the Radon transform. To accelerate this step, hierarchical mesh-grid algorithm is implemented. Once the axis position is known, localization of the electrode tip is performed. The method was tested on simulated images, on ultrasound images of a tissue mimicking phantom containing a metallic electrode, and on real ultrasound images from breast biopsy. The results indicate that the algorithm is robust with respect to variations in electrode position and speckle noise. Localization accuracy is of the order of hundreds of micrometers and is comparable to the ultrasound system axial resolution.
\end{abstract}

\section{INTRODUCTION}

$\mathrm{M}$ ANY medical surgical procedures consist of introducing a small surgical instrument, such as a needle or electrode, into biological tissue. In biopsy, tissue samples are taken from a particular region of the body by means of a thin needle [1]. In breast cancer therapy, a radioactive substance is injected near the tumor [2]. In the field of neurological research, the electrical activity of a specific group of neurons is recorded by a thin electrode needle [3]. For such procedures, it is often useful to know the instrument position during the intervention.

Manuscript received March 13, 2007; accepted December 26, 2007. M. Uherčík was supported by an EC project MEST-CT-2005-021024 WARTHE (Wide Applications Research Training in Health Engineering). J. Kybic was supported by the Czech Ministry of Education project MSM6840770012.

M. Barva, M. Uherčík, J. Kybic, and V. Hlaváč are with the Center for Machine Perception, Czech Technical University in Prague, Czech Republic (e-mail: uhercik@cmp.felk.cvut.cz).

M. Barva and M. Uherčík are also with and J.-M. Mari, H Liebgott, and Christian Cachard are with CREATIS, Université de Lyon, INSA-Lyon, Université Lyon 1, CNRS UMR5220, INSERM U630, France (e-mail: christian.cachard@creatis.univ-lyon1.fr).

J.-R. Duhamel is with Insitut des Sciences Cognitives, CNRS UMR 5229 Université de Lyon, Université Lyon 1, France.

Digital Object Identifier 10.1109/TUFFC.2008.833

\section{A. Existing Localization Techniques}

Horsley and Clarke [4], in 1908, introduced the stereotactic frame for instrument localization on small animals. They used a frame fixed with respect to external anatomical landmarks to place an electrode at a specific point in the animal's brain. However, the position of intracranial point often cannot be deduced from external landmarks with sufficient accuracy. To surmount this problem, stereotaxy is currently combined with magnetic resonance imaging (MRI) or computed tomography (CT) images. This technique permits reaching localization accuracy of the order of a tenth of a millimeter [5]. To avoid the limitations and patient discomfort associated with a stereotactic frame, frameless techniques were proposed. One of them is the spatial localization using a radio-frequency signal [6]. Three fixed orthogonal coils generate electromagnetic waves that are picked up by small coils attached to the tracked surgical instrument. Its position is estimated from the phase shift of received signals. Optical tracking with two calibrated cameras is also used [7]. The threedimensional (3-D) position of a marker on the tracked tool is determined from its projection in the images. The main difficulty is that the marker point must be visible in both cameras. Recently, tool localization using medical imaging modalities such as MRI, CT, and ultrasound was proposed [8]. They allow the physicians to view anatomical information of the human body as well as surgical instruments during the intervention. In terms of applicability to the problem of metallic instrument localization, the ultrasound imaging modality offers a number of advantages: short acquisition time permits real-time imaging, no ionizing radiation is involved, and compatibility with metallic objects. The resolution of modern systems is approximately $1 \mathrm{~mm}$. The purchase and operational cost is low compared to other medical imaging modalities.

\section{B. Problem Description}

The use of ultrasound for image-guidance procedures has become a clinical routine [8]. Glimcher [9] investigated the observability of a thin electrode inside a primate cortex in 2-D ultrasound images. From the experiments, he concludes that the ultrasound modality permits visualization of the anatomical structure together with a thin needle of diameter comparable to the ultrasound ax- 
ial resolution. However, observing a 3-D object using conventional B-mode images presents inherent limitations. In general, only a cross section is observable, unless the electrode axis is aligned with the scan plane. This is difficult to achieve without a special mechanical setup. To overcome this problem, a 3-D ultrasound scanner has been used instead [10], [17].

The task consists of automatic identification of a surgical instrument in the 3-D ultrasound image. The information about its position permits extraction from the acquired 3-D image of a planar section in which it is easier for a human to observe the tool. The position of the tool is determined in a coordinate system related to the ultrasound probe.

The task of an automatic object localization in ultrasound data is difficult because of attenuation, speckle noise, shadows, low contrast between the object and background, and signal dropouts. Large amounts of data require a fast algorithm to perform a real-time localization.

\section{Previous Work}

A variety of algorithms for object localization in ultrasound data have been proposed. The position of an axis of an object such as an electrode can be determined in a 2-D image using the principal component analysis (PCA) [11]. Initially, a variance image is computed as the intensity variance in a small neighborhood of each pixel of the original image. Next, the variance image is thresholded, and the PCA is applied to the covariance matrix of the coordinates of the thresholded pixels. The electrode axis is determined by the eigenvector corresponding to the largest eigenvalue. The experiments with a breast biopsy needle of $2.1 \mathrm{~mm}$ in diameter show that the intercept and the needle tip can be determined by the algorithm with accuracy of $1 \mathrm{~mm}$ for a depth of insertion greater than $15 \mathrm{~mm}$. Novotny et al. [12] enhanced this technique to 3-D images. Original image is segmented by thresholding, and compact clusters of voxels are identified. The cluster with the highest length to width ratio is selected. The experiment with a rod of 2-mm diameter was done. Tao et al. [13] introduced ultrasound image segmentation based on model-fitting. Geometry of the 3-D object to localize is described by a superquadric, whose parameters are estimated by a RANSAC (random sample consensus) estimator [14]. Barva et al. [15] using a polynomial curve model reported accuracy $0.2 \mathrm{~mm}$ for an electrode of diameter $0.5 \mathrm{~mm}$. Another approach is based on the observation that the electrode is more conspicuous in a projected image, and that its projection is minimized when the projection is performed along the electrode axis [16]. In the first step, the original image is projected to a plane. The direction of the electrode projection in the plane together with the projection direction determines a plane that contains the actual electrode. Adaptive 1-D search is used to minimize the projection area of the electrode. The reported accuracy is $0.4 \mathrm{~mm}$. To minimize the projected area of the electrode, Novotny et al. [18] uses a modified Radon transform. They divided the volume to smaller spherical regions and detected the axis locally using a fast method implemented on a graphics processing unit (GPU). They demonstrated a real-time tracking of tubular instruments of diameter $5 \mathrm{~mm}$ in a cardiac sequence with accuracy $0.2 \mathrm{~mm}$.

\section{Proposed Method}

We introduce an algorithm that permits automatic estimation of the position of a thin electrode in a 3-D ultrasound image. It identifies the electrode axis and its tip. The following assumptions about the electrode appearance in the image are made: (i) the electrode appears as a cylindrical object with a straight axis and a length much greater than a diameter, and (ii) the intensity of electrode voxels is much higher than the intensity of background voxels. Based on these hypotheses, the localization of electrode axis is done using a parallel projection. We formalize the 3-D projection into the form of a Parallel Integral Projection (PIP) transform and show that the electrode axis can be found by maximizing the PIP transform of the original image. The maximization is accelerated by means of a hierarchical mesh-grid algorithm. In a second phase, the electrode tip is found. This is accomplished by optimal thresholding that exploits prior probability densities of object and background voxel intensities.

The method presented here is designed for approximately straight needles, which covers most situations during intervention. For curved needles, other localization methods are intended, such as the method based on RANSAC and a polynomial curve model [15].

\section{A. Axis Localization with Parallel Integral Projection Transform}

The PIP is a transform that maps an image function $\mathcal{I}: \mathbb{R}^{3} \rightarrow \mathbb{R}$ representing volume data to a function $\mathcal{P}_{\mathcal{I}}: \mathbb{R}^{4} \rightarrow \mathbb{R}$ describing its projections as a function of the 2 -D displacement $(u, v)$ and the projection direction determined by two angles $(\alpha, \beta)$ [Fig. 1(a)]. More formally, the PIP transformation of $\mathcal{I}(\mathbf{x})$ is defined by an integral along a line passing through the point $Q=[u, v]$ with direction $\mathbf{w}$ given by angles $\alpha, \beta$ :

$$
\mathcal{P}_{\mathcal{I}}(u, v, \alpha, \beta)=\int_{-\infty}^{\infty} \mathcal{I}\left(\mathbf{R}(\alpha, \beta) \cdot(u, v, \tau)^{T}\right) \mathrm{d} \tau,
$$

where:

$$
\mathbf{R}(\alpha, \beta)=\left(\begin{array}{ccc}
\cos \beta & \sin \alpha \sin \beta & -\cos \alpha \sin \beta \\
0 & \cos \alpha & \sin \alpha \\
\sin \beta & -\sin \alpha \cos \beta & \cos \alpha \cos \beta
\end{array}\right)
$$

is the rotation matrix representing a rotation around the $x$-axis by angle $\alpha$, and around the $y$-axis by angle $\beta$. The PIP transform is a form of the Radon transform used in CT [19]. The same transform as PIP was called a modified Radon transform in [18]. The Radon transform usually is 


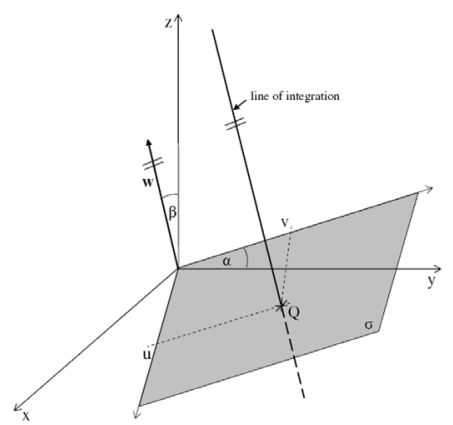

(a)

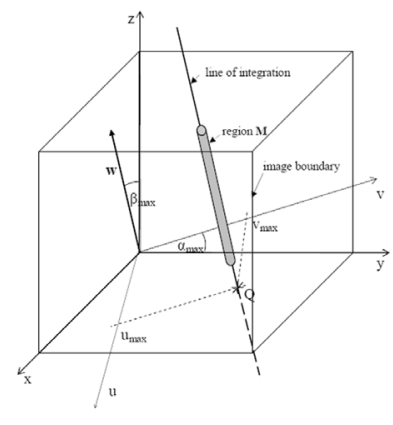

(b)
Fig. 1. (a) The PIP transform - the integral of the image intensity function $\mathcal{I}$ in (1) is calculated along a line given by the point $Q=$ $[u, v]$ and a vector $\mathbf{w}$. (b) Evaluation of the PIP transformation of a 3 -D image. Electrode is represented by a cylinder $M$. In this particular configuration, $\mathcal{P}_{\mathcal{I}}$ is maximized as the line of integration is identical with the electrode axis.

defined [20] as an integral of a 3-D function over parallel planes. The PIP transform is an integral over parallel lines. This transformation is sometimes also called an Xray transform.

The PIP transform can be used to identify the axis of a thin electrode in a 3-D image as follows [Fig. 1(b)]: Let $\mathcal{I}: \mathbb{R}^{3} \rightarrow \mathbb{R}$ be an image function such that the intensity of an electrode $\mathbf{M}$ is strictly greater than the background intensity:

$$
\forall \mathbf{x}_{1} \in \mathbf{M}, \forall \mathbf{x}_{2} \notin \mathbf{M}: \mathcal{I}\left(\mathbf{x}_{1}\right)>\mathcal{I}\left(\mathbf{x}_{2}\right) .
$$

Then, as the electrode diameter tends to zero, the location of the PIP maximum:

$$
\left(u_{\max }, v_{\max }, \alpha_{\max }, \beta_{\max }\right)=\arg \max \mathcal{P}_{\mathcal{I}}(u, v, \alpha, \beta),
$$

approaches the axis of $\mathbf{M}$ with a parametric equation:

$$
a(t)=\mathbf{R}\left(\alpha_{\max }, \beta_{\max }\right) \cdot\left(u_{\max }, v_{\max }, t\right)^{T} ; \forall t \in \mathbb{R} .
$$

1. Discretization of the PIP Transformation: We shall maximize the PIP transformation of a 3 -D image on a discrete grid. The discretization steps $\Delta_{\alpha}, \Delta_{\beta}, \Delta_{u}, \Delta_{v}$ must be sufficiently fine not to miss the electrode. We set them such that:

$$
\begin{aligned}
& \Delta_{\alpha}, \Delta_{\beta} \leq 2 \arctan \frac{d}{2\left\|\mathbf{x}_{\max }\right\|} \\
& \Delta_{u}, \Delta_{c} \leq d
\end{aligned}
$$

where $d$ is the electrode diameter and $\mathbf{x}_{\max }$ is the position of the most distant voxel from the origin. The motivation comes from the requirement that at least one integration line passes through the electrode (Fig. 2).

The integral $\mathcal{P}_{\mathcal{I}}$ is evaluated numerically. The integration line is sampled with a sampling step corresponding to the axial resolution of the ultrasound system. The integral is found using the trapezoidal rule.

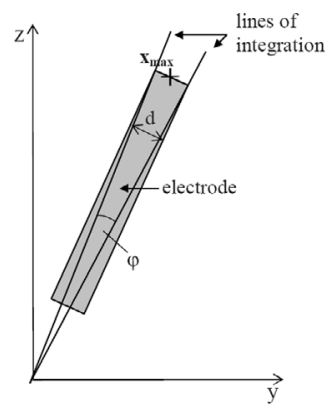

(a)

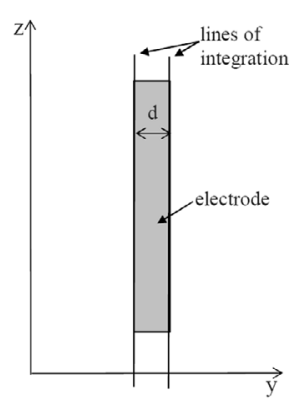

(b)
Fig. 2. (a) Discretization step of angles $\alpha, \beta$. If two lines of integration incline at angle $\varphi$ for which (6) holds, then at least one of the lines fully intersects the electrode. (b) Discretization step of variables $u, v$. Assuming two parallel lines of integration, the displacement must be less than the electrode diameter $d$, so that the electrode comprises at least one line of integration.

2. Maximizing the PIP Transformation: We decompose the maximization of $P_{I}(u, v, \alpha, \beta)$ to an inner maximization with respect to $(u, v)$ and an outer maximization with respect to $(\alpha, \beta)$.

Let us define a function:

$$
\mathcal{A}(\alpha, \beta)=\max _{u, v} \mathcal{P}_{\mathcal{I}}(u, v, \alpha, \beta),
$$

that will be referred to as the angle function. Angles $\alpha_{\max }, \beta_{\max }$ maximizing $\mathcal{A}(\alpha, \beta)$ also maximize $\mathcal{P}_{\mathcal{I}}(u, v, \alpha, \beta)$ for some $u_{\max }, v_{\max }$,

$$
\begin{gathered}
\exists u_{\max }, v_{\max }: \\
\left(u_{\max }, v_{\max }, \alpha_{\max }, \beta_{\max }\right)=\arg \max \mathcal{P}_{\mathcal{I}} \Leftrightarrow \\
\Leftrightarrow\left(\alpha_{\max }, \beta_{\max }\right)=\arg \max \mathcal{A} .
\end{gathered}
$$

To determine the value of $\mathcal{A}(\alpha, \beta)$, we first find the circumscribed rectangle $\left\langle u_{1}, u_{2}\right\rangle \times\left\langle v_{1}, v_{2}\right\rangle$ of the parallel projection of the image boundary onto the plane $\sigma$ by a normal vector $\mathbf{w}=\mathbf{R}(\alpha, \beta) \cdot(1,0,0)^{T}$ (Fig. 3$)$ as:

$$
\begin{aligned}
& u_{1}=\min _{\mathbf{x} \in \Omega}\left(\mathbf{R}^{-1}(\alpha, \beta) \cdot \mathbf{x}\right) \cdot \mathbf{e}_{x}, \\
& u_{2}=\max _{\mathbf{x} \in \Omega}\left(\mathbf{R}^{-1}(\alpha, \beta) \cdot \mathbf{x}\right) \cdot \mathbf{e}_{x}, \\
& v_{1}=\min _{\mathbf{x} \in \Omega}\left(\mathbf{R}^{-1}(\alpha, \beta) \cdot \mathbf{x}\right) \cdot \mathbf{e}_{y}, \\
& v_{2}=\max _{\mathbf{x} \in \Omega}\left(\mathbf{R}^{-1}(\alpha, \beta) \cdot \mathbf{x}\right) \cdot \mathbf{e}_{y} .
\end{aligned}
$$

where $\Omega$ is a set of voxel coordinates and $\mathbf{e}_{x}, \mathbf{e}_{y}$ are unit vectors in the Cartesian coordinate system. The integral in (1) is evaluated at a grid of points $(u, v)$ uniformly distributed on the rectangle $\left\langle u_{1}, u_{2}\right\rangle \times\left\langle v_{1}, v_{2}\right\rangle$ with steps $\Delta_{u}$, $\Delta_{v}$ satisfying (7). The value of $\mathcal{A}(\alpha, \beta)$ is the maximum of $\mathcal{P}_{\mathcal{I}}$ exhaustively evaluated on the grid with fixed $\alpha, \beta$.

We shall now find values $\alpha_{\max }, \beta_{\max }$ that maximize the angle function $\mathcal{A}(\alpha, \beta)$ :

$$
\left(\alpha_{\max }, \beta_{\max }\right)=\arg \max \mathcal{A}(\alpha, \beta) .
$$

Two approaches to this maximization have been tested: exhaustive search, and hierarchical mesh-grid search. 


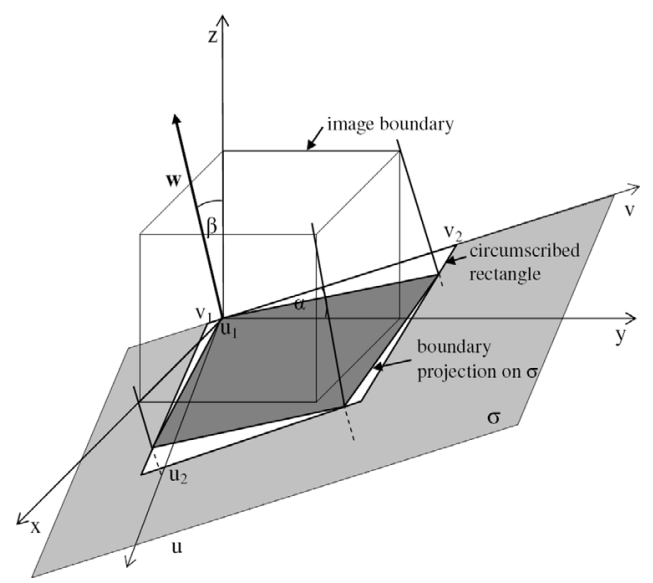

Fig. 3. Evaluation of the angle function $\mathcal{A}(\alpha, \beta)$. 3-D image boundary is projected on the plane $\sigma$ and a circumscribed rectangle comprising the projection is determined. Sampling the rectangle with a uniform grid of points $(u, v)$, the value of $\mathcal{A}(\alpha, \beta)$ is taken to be equal to $\max _{u, v} \mathcal{P}_{\mathcal{I}}(\alpha, \beta, u, v)$ evaluated on this grid for fixed $(\alpha, \beta)$.

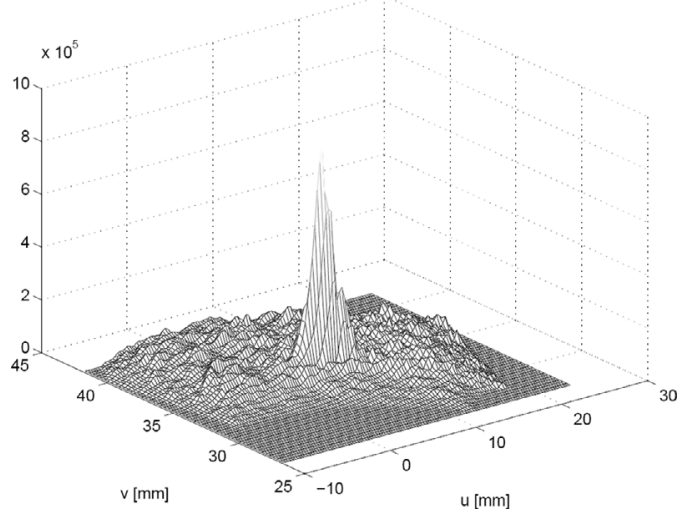

Fig. 4. PIP transformation of a 3-D image for a given angles $(\alpha, \beta)$. You can see the peak in the middle representing the high response of the electrode.

Other approaches, such as gradient descent algorithm, are also possible [21]. We decided to use the hierarchical meshgrid method because it does not require an initial solution and does not need derivates and to some extent can avoid a local maximum.

a) Exhaustive search: Due to the periodicity of the PIP transform in $\alpha, \beta$, it suffices to maximize the angle function in the interval $\left\langle 0^{\circ}, 180^{\circ}\right\rangle \times\left\langle 0^{\circ}, 180^{\circ}\right\rangle$. This grid is uniformly sampled with discretization steps satisfying (6), (7), and the angle function is evaluated at each grid point (Figs. 4 and 5).

b) Hierarchical mesh-grid search: The main drawback of the exhaustive search is its computational complexity. To alleviate it, we propose to use the hierarchical meshgrid search method [22]. On the first level, $\mathcal{A}(\alpha, \beta)$ is evaluated on a rectangular grid of points $\left\langle 0^{\circ}, 180^{\circ}\right\rangle \times\left\langle 0^{\circ}, 180^{\circ}\right\rangle$ that are uniformly sampled with steps $\Delta_{\alpha}^{\text {init }}, \Delta_{\beta}^{\text {init }}$ (Fig. 6). The maximum location $\left(\alpha_{\max }^{1}, \beta_{\max }^{1}\right)$ is determined. On the second level, the angle function is evaluated on a rectangular grid $\left\langle\alpha_{\max }^{1}-45^{\circ}, \alpha_{\max }^{1}+45^{\circ}\right\rangle \times\left\langle\beta_{\max }^{1}-45^{\circ}\right.$,

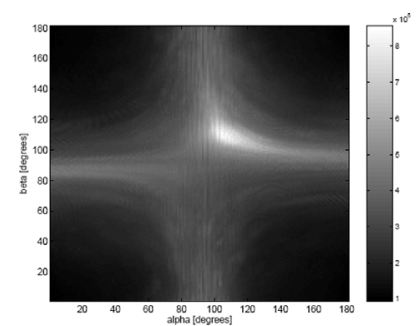

(a)

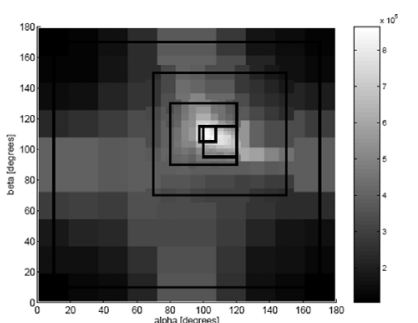

(b)
Fig. 5. Maximization of the angle function $\mathcal{A}(\alpha, \beta)$. (a) Exhaustive search with discretization steps evaluates $\mathcal{A}(\alpha, \beta)$ at each grid point of a square grid $\left\langle 0,180^{\circ}\right\rangle \times\left\langle 0,180^{\circ}\right\rangle$ uniformly sampled with discretization steps $\Delta_{\alpha}=\Delta_{\beta}=1^{\circ}$. (b) Five-level hierarchical meshgrid search with initial steps $\Delta_{\alpha}^{1}=\Delta_{\beta}^{1}=16^{\circ}$ and final discretization steps $\Delta_{\alpha}^{5}=\Delta_{\beta}^{5}=1^{\circ}$. The size of a search region and discretization steps $\Delta_{\alpha}, \Delta_{\beta}$ are decreased by a factor of 2 at each level. Black rectangles in the figure delineate the region of search on each level.

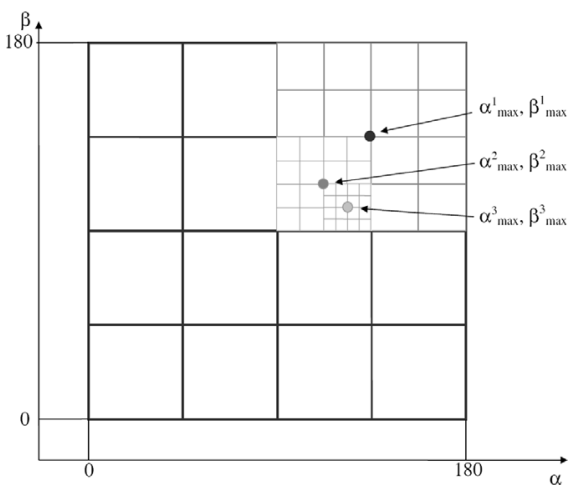

Fig. 6. Principle of hierarchical mesh-grid algorithm applied to maximum search of the angle function $\mathcal{A}(\alpha, \beta)$. On the first level, the angle function $\mathcal{A}$ is evaluated on a grid of points and $\alpha_{\max }^{1}, \beta_{\text {max }}^{1}$ is selected. On the second level, the $\mathcal{A}$ is evaluated on a grid of points with a smaller range and a smaller step, then $\alpha_{\max }^{2}, \beta_{\max }^{2}$ is selected, etc.

$\left.\beta_{\max }^{1}+45^{\circ}\right\rangle$ uniformly sampled with steps $\Delta_{\alpha}^{2}=\left(\Delta_{\alpha}^{1} / 2\right)$, $\Delta_{\beta}^{2}=\left(\Delta_{\beta}^{1} / 2\right)$, where $\Delta_{\alpha}^{1}=\Delta_{\alpha}^{\text {init }}, \Delta_{\beta}^{1}=\Delta_{\beta}^{\text {init }}$. The pair $\left(\alpha_{\text {max }}^{2}, \beta_{\text {max }}^{2}\right)$ that maximizes $\mathcal{A}(\alpha, \beta)$ is established. Generally, on the $i$-th level, the angle function is evaluated on a grid $\left\langle\alpha_{\max }^{i-1}-\left(180^{\circ} / 2^{i}\right), \alpha_{\max }^{i-1}+\left(180^{\circ} / 2^{i}\right)\right\rangle \times$ $\left\langle\beta_{\max }^{i-1}-\left(180^{\circ} / 2^{i}\right), \beta_{\max }^{i-1}+\left(180^{\circ} / 2^{i}\right)\right\rangle$ with discretization steps $\left.\Delta_{\alpha}^{i}=\left(\Delta_{\alpha}^{1} / 2^{i-1}\right), \Delta_{\beta}^{i}=\left(\Delta_{\beta}^{1} / 2^{i-1}\right)\right\rangle$. The algorithm continues until both steps $\Delta_{\alpha}^{i}, \Delta_{\beta}^{i}$ are equal, or inferior to some predefined threshold values $\Delta_{\alpha}^{\text {final }}, \Delta_{\beta}^{\text {final }}$ that control accuracy of axis localization.

c) Comparison of exhaustive search and hierarchical search: The main interest in using the hierarchical meshgrid search method is to accelerate the task of the PIP maximization. Let us compare the computational efficiency of both search methods in terms of the number of evaluations of $\mathcal{A}(\alpha, \beta)$. This number is influenced by the discretization parameters $\Delta_{\alpha}, \Delta_{\beta}$, resp. $\Delta_{\alpha}^{\text {init }}, \Delta_{\beta}^{\text {init }}$, $\Delta_{\alpha}^{\text {final }}, \Delta_{\beta}^{\text {final }}$ of the exhaustive, resp. hierarchical meshgrid method. 


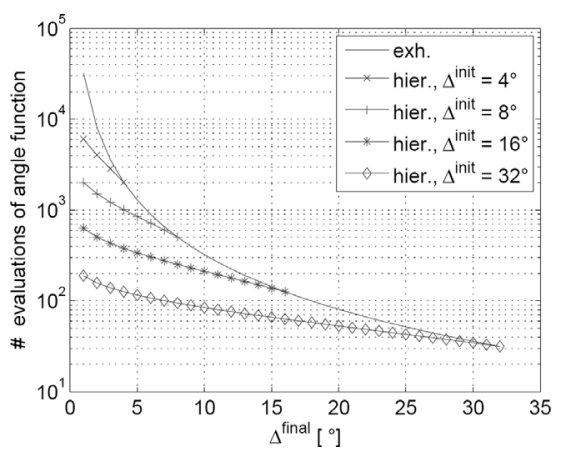

Fig. 7. Comparison of the number of evaluations of $\mathcal{A}(\alpha, \beta)$ as a function of discretization steps $\Delta_{\text {exh }}=\Delta^{\text {final }}$ for exhaustive and hierarchical mesh-grid searches.

Exhaustive search:

To determine the maximum of the function $\mathcal{A}(\alpha, \beta)$ with accuracy $\Delta_{\alpha}, \Delta_{\beta}$, the rectangle $\left\langle 0^{\circ}, 180^{\circ}\right\rangle \times$ $\left\langle 0^{\circ}, 180^{\circ}\right\rangle$ is sampled along dimensions $\alpha$, resp. $\beta$ dimension with the step $\Delta_{\alpha}$, resp. $\Delta_{\beta}$. The total number of the angle function evaluations is equal to:

$$
\frac{180^{\circ}}{\Delta_{\alpha}} \cdot \frac{180^{\circ}}{\Delta_{\beta}} \text {. }
$$

Hierarchical mesh-grid search:

On each level, the discretization steps are divided by a factor of 2. Given the initial $\Delta_{\alpha}^{\text {init }}, \Delta_{\beta}^{\text {init }}$, and final steps $\Delta_{\alpha}^{\text {final }}, \Delta_{\beta}^{\text {final }}$, the number of levels is:

$$
\left\lceil N=\max \left\{\log _{2} \frac{2 \Delta_{\alpha}^{\text {init }}}{\Delta_{\alpha}^{\text {final }}}, \log _{2} \frac{2 \Delta_{\beta}^{\text {init }}}{\Delta_{\beta}^{\text {final }}}\right\}\right\rceil .
$$

There are $\left(180 / \Delta_{\alpha}^{\text {init }}\right) \cdot\left(180 / \Delta_{\beta}^{\text {init }}\right)$ evaluations at each level, so the total number of function $\mathcal{A}(\alpha, \beta)$ evaluations performed by the hierarchical mesh-grid method is:

$$
N \cdot\left(\frac{180}{\Delta_{\alpha}^{\text {init }}} \cdot \frac{180}{\Delta_{\beta}^{\text {init }}}\right) .
$$

In order to simplify the comparison, discretization steps of both methods are assumed to be equal: $\Delta_{\alpha}=\Delta_{\beta}=$ $\Delta_{\alpha}^{\text {final }}=\Delta_{\beta}^{\text {final }}=\Delta^{\text {final }}, \Delta_{\alpha}^{\text {init }}=\Delta_{\beta}^{\text {init }}=\Delta^{\text {init }}$. Fig. 7 shows the number of evaluations of $\mathcal{A}(\alpha, \beta)$ as a function of $\Delta_{\alpha}=\Delta_{\beta}=\Delta^{\text {final }}$, where $\Delta^{\text {init }}$ is used as a parameter. We observe that using a hierarchical mesh-grid method leads to a significant decrease in computational cost.

\section{B. Electrode Tip Localization}

Once the electrode axis $a(t)$ is identified, we determine the coordinates of electrode endpoints. In most cases, the electrode is not entirely comprised in the field of view as only a small part of tissue is scanned by the scanning device. Therefore, we localize only one electrode endpoint (referred to as electrode tip) that is located inside the field of view.

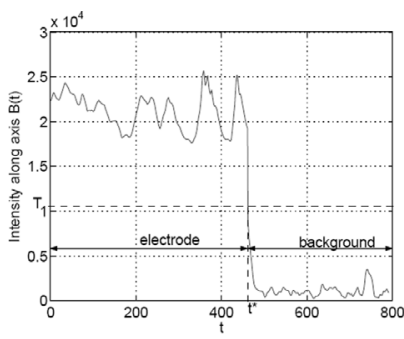

(a)

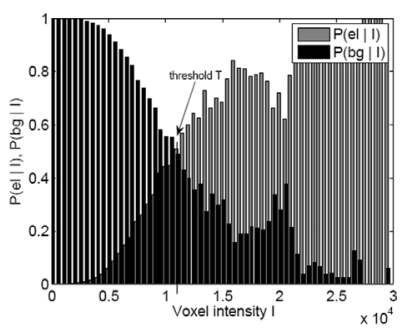

(b)
Fig. 8. (a) Intensity profile along the estimated axis $a(t)$ is used to identify the part of the axis that passes through the electrode. (b) Histograms of electrode and background voxels intensities are calculated a priori to approximate the conditional probabilities $P(e l \mid I)$, $P(b g \mid I)$.

Let $\mathcal{B}(t)$ be the voxel intensity along the estimated axis $a(t)(5)$ :

$$
\mathcal{B}(t)=\mathcal{I}(a(t)) ; \quad \forall t \in \mathbb{R}: a(t) \in \mathbf{\Omega} .
$$

Fig. 8 shows an example of an intensity profile along the estimated axis. While tracing the values of $\mathcal{B}(t)$ for increasing $t$, let $t^{*}$ be the first value where $\mathcal{B}(t)$ decreases under a predetermined threshold $T$. The coordinates of the electrode tip are given by $a\left(t^{*}\right)$.

The threshold value $T$ is determined from two a priori estimated probability distributions: probability of the electrode voxel $P(e l \mid I)$, resp. background voxel $P(b g \mid I)$ given the voxel intensity $I$. Parameter $T$ is such that $P(e l \mid T)=P(b g \mid T)$. To estimate these distributions, voxels were classified as electrode or background voxels in an acquired dataset with known electrode position.

\section{EXPERIMENTS}

Two types of 3-D ultrasound images were used to test the algorithm robustness: numerical phantoms simulated using the FIELD II software package [23], and real ultrasound images acquired with a $3-\mathrm{D}$ ultrasound scanner. The localization algorithm is implemented in MATLAB (The MathWorks, Natick, MA). All tests were performed on a Linux computer with a 64 -bit Intel Core 2 processor at $2400 \mathrm{MHz}$.

\section{A. Accuracy Assessment}

Two measures are used to quantify the accuracy of the proposed method. The first measure $\varepsilon_{\text {tip }}$ evaluates the tip localization accuracy:

$$
\varepsilon_{\text {tip }}=\|T-\hat{T}\|
$$

where $T$ is the true electrode tip, $\hat{T}$ is an estimated tip, and $\|\cdot\|$ is the Euclidean distance. Axis localization accuracy $\varepsilon_{\text {axis }}$ is given by:

$$
\varepsilon_{\text {axis }}=\max \left\{\left\|E-Q_{1}\right\|,\left\|T-Q_{2}\right\|\right\},
$$




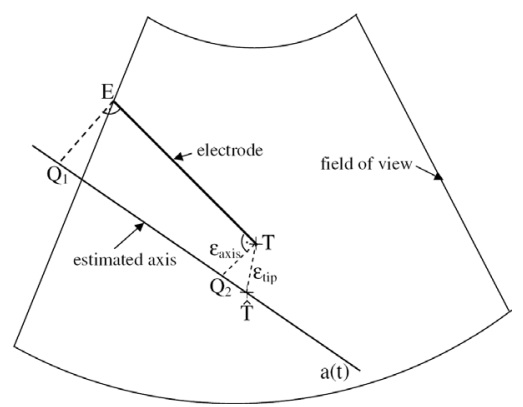

Fig. 9. Illustration of axis and endpoint accuracy evaluation. Groundtruth electrode position is determined by the intercept point $E$ and electrode tip $T$. We compare it with an axis $a(t)$ and a tip $\hat{T}$ estimated by the proposed method.

TABLE I

Parameters of Field II Used for Simulation of Numerical Phantoms.

\begin{tabular}{|c|c|}
\hline Transducer type & Linear array \\
\hline Number of elements & 128 \\
\hline Element width [mm] & 0.4 \\
\hline Element height $[\mathrm{mm}]$ & 5 \\
\hline $\operatorname{Kerf}[\mathrm{mm}]$ & 0.025 \\
\hline Initial focus $[\mathrm{mm}]$ & {$\left[\begin{array}{lll}0 & 0 & 55\end{array}\right]$} \\
\hline Central frequency $[\mathrm{MHz}]$ & 7.5 \\
\hline Sampling frequency $[\mathrm{MHz}]$ & 27 \\
\hline
\end{tabular}

where $E$ is the intercept point, $Q_{1}$ are $Q_{2}$ are the orthogonal projections of $E$ and $T$ on $a(t)$ with respect to the true axis, and $a(t)$ is the estimated axis (Fig. 9).

\section{B. Numerical Phantom}

Simulated data were generated using the ultrasound simulator FIELD II [23]. The simulator parameters were set to imitate the ultrasound scanner Voluson 530D (GE Healthcare, UK). We used a multi-element transducer to produce in total 53 sector scan planes with B-mode angle $40^{\circ}$, each of them composed of 71 scan lines. These planes were angularly distributed over a tilt angle of $40^{\circ}$ to scan a 3-D region. Table I summarizes the parameters set in FIELD II. The dimensions of the point spread function of the ultrasound system were set to $0.2 \mathrm{~mm}$ in axial and $1 \mathrm{~mm}$ in both lateral and azimuthal directions.

We simulated a series of numerical phantoms representing a cuboid region of tissue containing a highly reflecting inclusion corresponding to a metallic electrode. The dimensions of the tissue region were $50 \times 50 \times 30 \mathrm{~mm}$. and its axial distance from the probe was set to $35 \mathrm{~mm}$. To approximate the speckle pattern of biological tissue, the spatial density of tissue diffusers was set to 3 per $\mathrm{mm}^{3}$. Their reflection coefficients had normal distribution with zero mean and variance equal to 1 [24]. The electrode was represented by a cylindrical region of $0.3 \mathrm{~mm}$ in diameter and $20 \mathrm{~mm}$ in length containing diffusers with spatial density of 125 per $\mathrm{mm}^{3}$. Their reflection coefficients were constant and equal to 5. Fig. 10 depicts an example of a

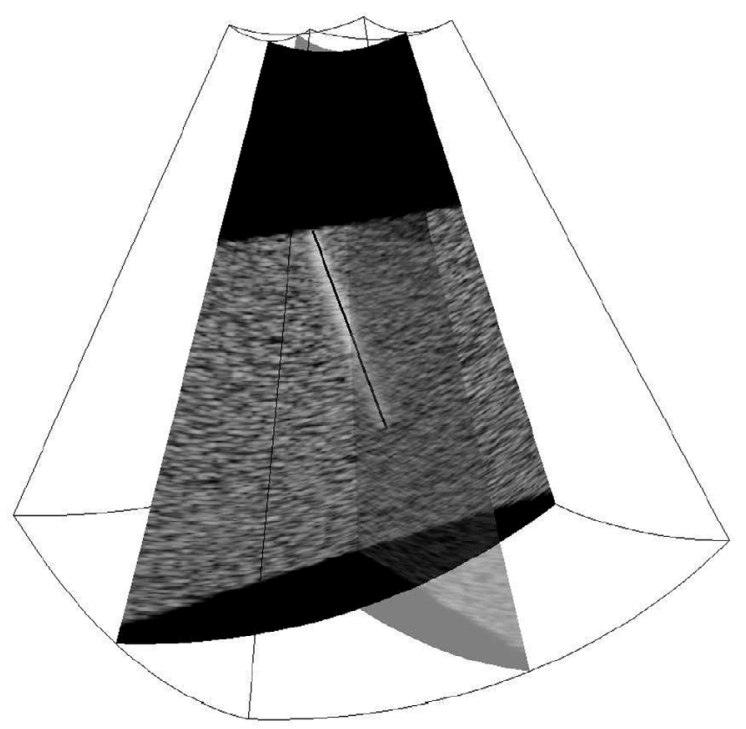

Fig. 10. Example of a 3-D numerical image simulated in FIELD II. It represents a portion of biological tissue containing a highly scattering inclusion such as electrode. Two planar sections are shown in grayscale; one of them is passing through the electrode axis. Black line-segment depicts electrode position estimated by the proposed method.

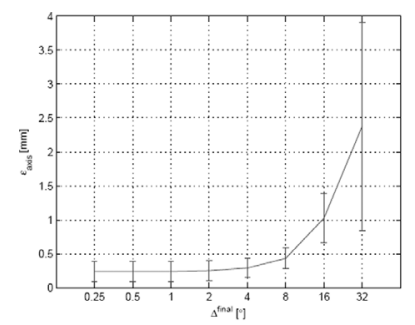

(a)

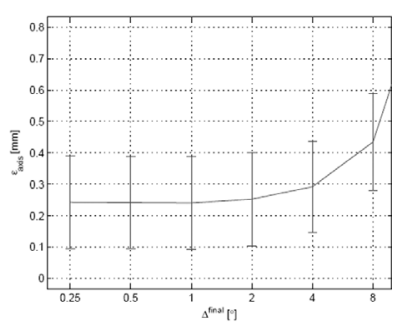

(b)
Fig. 11. Achieved localization accuracy $\varepsilon_{\text {axis }}$ for different values of the final angular step $\Delta^{\text {final }}$ with standard deviation shown with vertical bars. (a) A full chart. (b) A zoomed part for smaller $\Delta^{\text {final }}$ values.

3-D numerical phantom simulated in FIELD II. The exact knowledge of electrode axis and tip location is used to evaluate the algorithm accuracy.

1. Influence of Angular Step Size: The accuracy of the PIP method is good if the angular step parameter $\Delta^{\text {final }}$ is small. However, small $\Delta^{\text {final }}$ leads to an increase in computational time. To find the optimal value of $\Delta^{\text {final }}$, we performed 28 tests on a numerical phantom described in the previous paragraph. The electrode axis position was estimated by the algorithm while varying $\Delta^{\text {final }}$ from $0.125^{\circ}$ to $32^{\circ}$. Other parameters were constant: $\Delta^{\text {init }}=32^{\circ}$, and $\Delta_{u}=\Delta_{v}=0.2 \mathrm{~mm}$.

Fig. 11 illustrates axis localization accuracy $\varepsilon_{\text {axis }}$ as a function of $\Delta^{\text {final }}$. We conclude that decreasing this parameter under $1^{\circ}$ leads to an increase in computational time (Fig. 12) without further improvement in axis localization accuracy. We set $\Delta^{\text {final }}$ to $1^{\circ}$. 


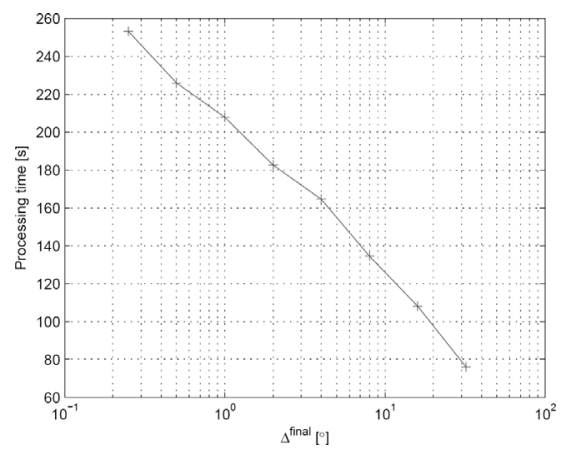

Fig. 12. Time required to maximize the PIP transformation for varying $\Delta^{\text {final }}$ with other parameters constant.

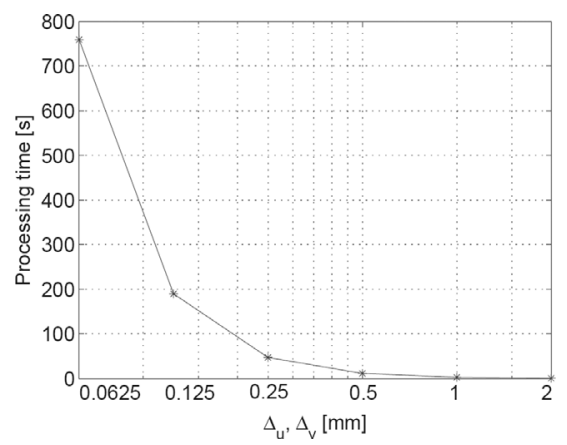

Fig. 13. Time required to maximize the PIP transformation for varying $\Delta_{u}, \Delta_{v}$ with other parameters constant.

2. Influence of Displacement Steps $\Delta_{u}, \Delta_{v}$ : The displacement parameters $\Delta_{u}, \Delta_{v}$ control the distance between adjacent lines of integration. While decreasing these parameters improves accuracy, it also increases the computational time (Fig. 13). The same numerical phantom as in Section III-B, 1 was used to experimentally determine optimum values of $\Delta_{u}, \Delta_{v}$. The localization algorithm was executed with fixed parameters $\Delta^{\text {init }}=32^{\circ}, \Delta^{\text {final }}=1^{\circ}$ and varying the displacement steps $\Delta_{u}, \Delta_{v}$ from 0.0625 to $2 \mathrm{~mm}$. Fig. 14 depicts the calculated axis accuracy $\varepsilon_{\text {axis }}$ as a function of the displacement steps $\Delta_{u}, \Delta_{v}$. It shows that, when $\Delta_{u}, \Delta_{v}$ exceeds $0.25 \mathrm{~mm}$, the mean and standard deviation of error in axis localization increases rapidly. We propose to set $\Delta_{u}, \Delta_{v}$ to $0.2 \mathrm{~mm}$.

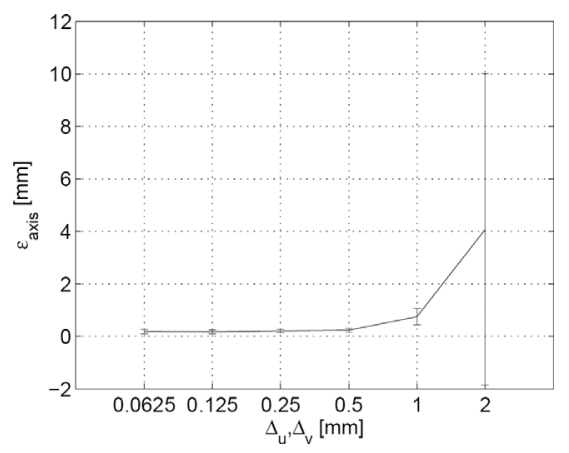

Fig. 14. Axis localization accuracy $\varepsilon_{\text {axis }}$ as a function of discretization steps $\Delta_{u}, \Delta_{v}$. Vertical bars in each node show the standard deviation.

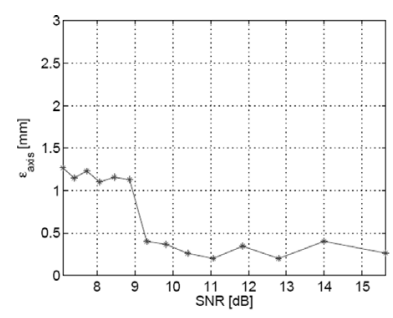

(a)

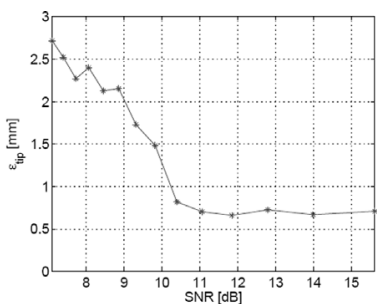

(b)
Fig. 15. Axis (a) and tip (b) localization accuracy as a function of image SNR.

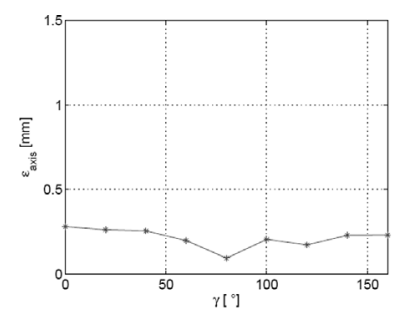

(a)

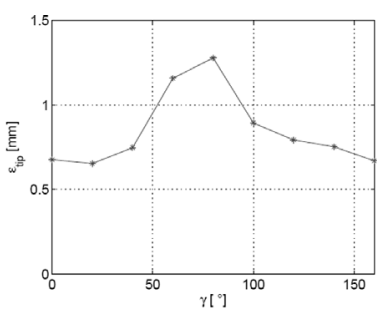

(b)
Fig. 16. Axis (a) and tip (b) localization accuracy as a function of electrode orientation with respect to probe axis.

3. Effect of Background Noise: In real applications, the intensity of electrode and background voxels varies randomly. To investigate the influence of background noise, we simulated 14 numerical phantoms with the electrode at the same position while increasing the background noise. Given the mean $\mu_{b g}$ and the variance $\sigma_{b g}^{2}$ of background voxels and the mean $\mu_{e l}^{2}$ and the variance $\sigma_{e l}$ of electrode voxels, we define the signal-to-noise ratio (SNR) as the logarithm of the expected energy of electrode voxels to expected energy of background voxel intensities,

$$
\mathrm{SNR}=10 \log \frac{\mu_{e l}^{2}+\sigma_{e l}^{2}}{\mu_{b g}^{2}+\sigma_{b g}^{2}}[\mathrm{~dB}] .
$$

The value of SNR is used to quantify the ratio of electrode intensity to background noise. The electrode axis was localized in each phantom with fixed parameters: $\Delta^{\text {init }}=32^{\circ}, \Delta^{\text {final }}=1^{\circ}$ and $\Delta_{u}=\Delta_{v}=0.2 \mathrm{~mm}$.

Experimental dependences of $\varepsilon_{\text {axis }}, \varepsilon_{\text {tip }}$ on SNR are depicted in Fig. 15. When background noise was increased, we observed a decrease in both axis and tip localization accuracy. The axis localization error grows rapidly for SNR inferior to $9 \mathrm{~dB}$.

4. Varying Electrode Orientation and Position: The angle $\gamma$ between the electrode axis and the probe axis was varied from $0^{\circ}$ to $160^{\circ}$. The electrode axis and tip were localized in each phantom using our localization algorithm with the same settings as in Section III-B,3.

Localization accuracy $\varepsilon_{\text {axis }}, \varepsilon_{\text {tip }}$ are shown in Fig. 16 as a function of electrode orientation. Note that axis accuracy $\varepsilon_{\text {axis }}$ is high for $\gamma=90^{\circ}$, whereas the tip accuracy $\varepsilon_{\text {tip }}$ is low. This is a consequence of the anisotropic spatial resolution of the ultrasound system. 
TABLE II

Achieved Localization Accuracy on Numerical Phantoms with Spherical Artifacts.

\begin{tabular}{lcccccccccc}
\hline \#image & 1 & 2 & 3 & 4 & 5 & 6 & 7 & 8 & 9 & mean \\
\hline Spheres & 2 & 2 & 2 & 3 & 3 & 5 & 5 & 6 & 6 & 3.44 \\
SNR [dB] & 10.03 & 11.87 & 15.02 & 8.46 & 10.44 & 7.17 & 16.01 & 11.51 & 15.02 & 11.72 \\
$\varepsilon_{\text {axis }}[\mathrm{mm}]$ & 0.097 & 0.210 & 0.097 & 0.210 & 0.166 & 0.185 & 0.187 & 0.097 & 0.097 & 0.150 \\
$\varepsilon_{\text {tip }}[\mathrm{mm}]$ & 0.497 & 0.067 & 0.282 & 0.074 & 0.090 & 1.068 & 0.308 & 0.282 & 0.282 & 0.328 \\
Time [sec] & 678 & 681 & 679 & 674 & 920 & 670 & 895 & 674 & 915 & 754 \\
\hline
\end{tabular}

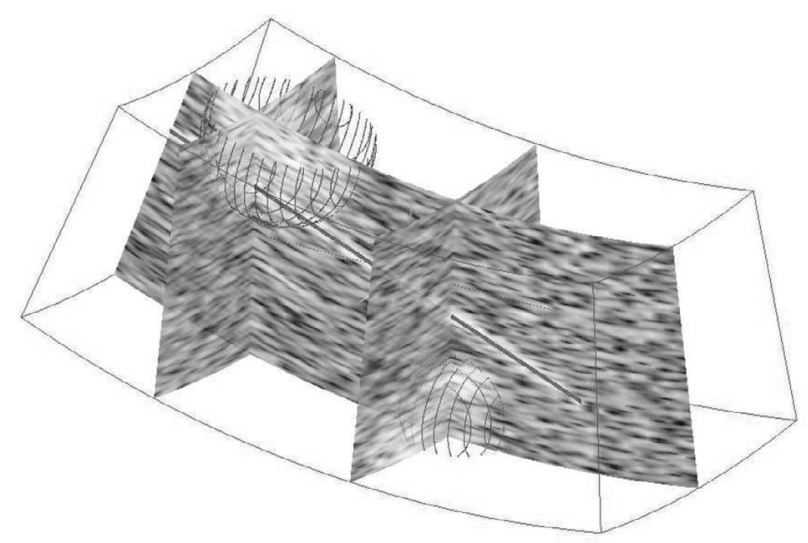

Fig. 17. Example of a numerical phantom with two spherical artifacts shown as wireframe spheres (with higher reflection coefficients). We also show a planar cross section containing the electrode (marked with line) and two perpendicular planar sections.

5. Nonhomogenous Background: We created a numerical phantom with spherical artifacts to imitate the inhomogenity of real biological tissue. The spheres were randomly placed, so they might overlap each other or the electrode. The number of spheres varied between two and six, the radius varied between 2 and $5 \mathrm{~mm}$. The spatial density of diffusers in spheres was 2.5 per $\mathrm{mm}^{3}$. Their reflection coefficient varied randomly between 2 and 5 . Fig. 17 depicts an example of such a phantom (dataset number 1 in Table II). The exact knowledge of axis and tip locations was used to evaluate the algorithm accuracy.

The PIP algorithm succeeded to localize the electrode axis in all datasets. For each dataset accuracy of axis and tip of electrode was evaluated and also SNR was computed. The results are in the Table II. The mean accuracy of the axis localization is $0.150 \mathrm{~mm}$. The mean accuracy of tip localization $0.328 \mathrm{~mm}$ was similar to homogenous numerical phantoms.

\section{Cryogel Phantom}

The algorithm was tested on real 3-D ultrasound data as well. To simulate biological tissue with a highly reflecting inclusion, a polyvinyl alcohol (PVA) cryogel phantom [25] with dimensions $50 \times 50 \times 50 \mathrm{~mm}$ was used. Inside the phantom there was a thin tungsten electrode of $150 \mu \mathrm{m}$ in diameter and length $20 \mathrm{~mm}$ (Fig. 18).

The phantom was scanned with a 3-D ultrasound scanner Voluson 530D that was modified to provide RF data

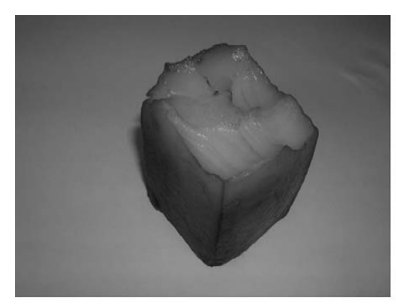

(a)

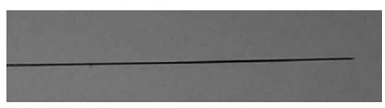

(b)
Fig. 18. (a) Cuboidal of polyvinyl alcohol (PVA) cryogel phantom mimicking acoustic properties of biological tissue. The dimensions are $50 \times 50 \times 50 \mathrm{~mm}$. (b) Tungsten electrode of $150 \mu \mathrm{m}$ in diameter and length $20 \mathrm{~mm}$ was introduced into the phantom prior to scanning.

[26]. The scanner was equipped with the S-VDW5-8B3D Voluson probe operating at central frequency $7.5 \mathrm{MHz}$. Tilt angle was set to $40^{\circ}$ at interval $0.75^{\circ}$. The angle of acquired B-mode sector images was $40^{\circ}$ with the angular step $0.5^{\circ}$. The phantom orientation and distance from the probe was slightly varied between scanning to obtain eight 3 -D images of $53 \times 71 \times 3100$ voxels (Fig. 19). The axial resolution was $0.4 \mathrm{~mm}$ and the lateral resolution was approximately $1 \mathrm{~mm}$.

To evaluate accuracy $\varepsilon_{\text {axis }}$ and $\varepsilon_{\text {tip }}$, the intercept and electrode tip coordinates were manually determined in each dataset and considered as the ground-truth. The boundary of phantom was excluded from the region of interest so the input for algorithm consisted of only the interior of the phantom. Table III summarizes localization accuracy for each ultrasound image.

The mean computational time was 18.7 minutes for one volume dataset. The mean accuracy on datasets with mean SNR $17.7 \mathrm{~dB}$ was $0.301 \mathrm{~mm}$ for the electrode axis and $0.263 \mathrm{~mm}$ for the tip, which is satisfactory.

\section{Breast Biopsy}

To demonstrate the usability of PIP on real biological tissue, we ran the algorithm on data from breast biopsy (Fig. 20). This dataset was taken from 3-D ultrasound scanner GE Voluson E8 with the probe operating at a central frequency of $12 \mathrm{MHz}$. The 3-D image was computed from sliding B-mode sector images at interval $0.1 \mathrm{~mm}$, the total width of the volume was $38 \mathrm{~mm}$. The angle of acquired B-mode sector images was $30^{\circ}$ with an angular step of $0.1448^{\circ}$. The volume depth was $19 \mathrm{~mm}$. Inside the breast there was a 19-gauge needle (1.092 $\mathrm{mm}$ outer diameter) made of stainless steel. 
TABLE III

Achieved Localization Accuracy on Real Ultrasound Images.

\begin{tabular}{lccccccccc}
\hline \#image & 1 & 2 & 3 & 4 & 5 & 6 & 7 & 8 & mean \\
\hline$\varepsilon_{\text {axis }}[\mathrm{mm}]$ & 0.218 & 0.663 & 0.323 & 0.157 & 0.287 & 0.200 & 0.442 & 0.118 & 0.301 \\
$\varepsilon_{\text {tip }}[\mathrm{mm}]$ & 0.120 & 0.003 & 0.283 & 0.174 & 0.450 & 0.463 & 0.370 & 0.245 & 0.263 \\
SNR [dB] & 17.9 & 18.1 & 17.6 & 19.1 & 14.6 & 16.8 & 18.6 & 19 & 17.7 \\
Time [sec] & 451 & 309 & 1865 & 1727 & 393 & 779 & 1846 & 1595 & 1121 \\
\hline
\end{tabular}

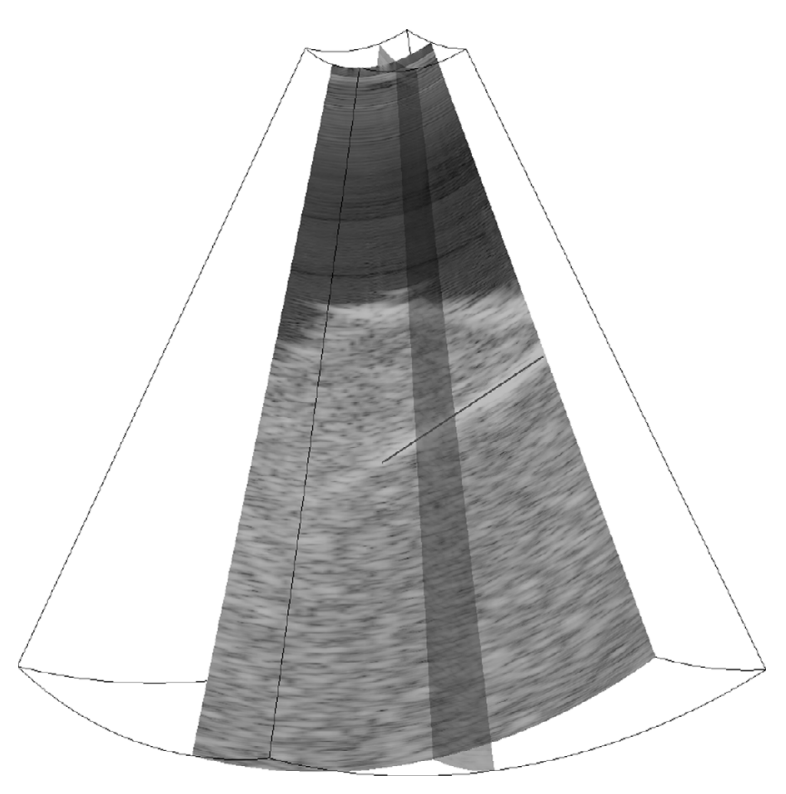

Fig. 19. Example of 3-D ultrasound image of PVA cryogel phantom submerged in water. Inside the phantom there was a tungsten electrode. Twoplanar sections (one of them passing through the electrode axis) are shown in grayscale. Black line-segment represents estimated electrode position.

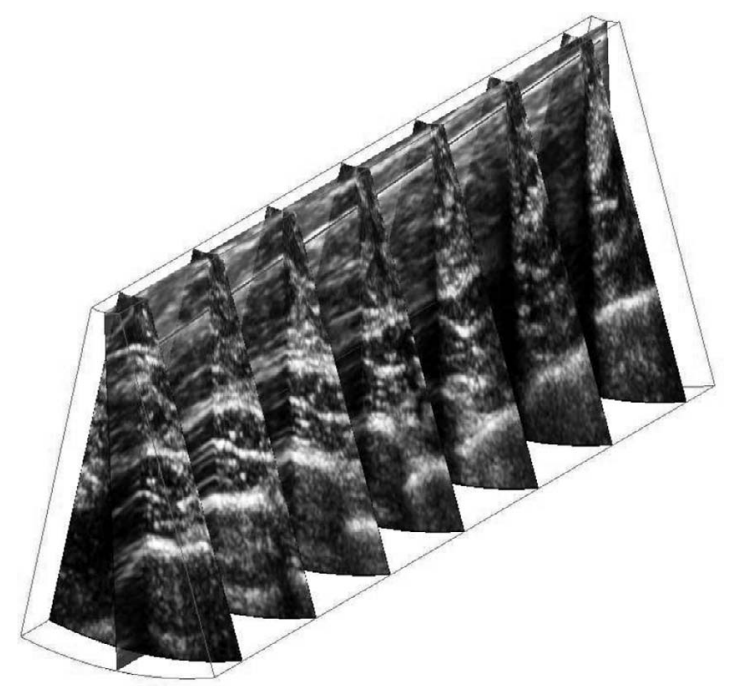

Fig. 20. 3-D view of data from breast biopsy. The boundary geometry of 3-D data is marked by thin lines. There is one planar section with a needle in the upper part and seven perpendicular planar sections.
To evaluate accuracy $\varepsilon_{\text {axis }}$, the electrode was manually localized in the dataset and the position was considered as the ground-truth. We obtained axis localization accuracy $0.193 \mathrm{~mm}$, which is similar to results on phantom images. We could not evaluate the electrode tip accuracy because the tip was located outside the image. Our method performed satisfactorily, even though the SNR of this dataset was only $5.43 \mathrm{~dB}$, which is typical for real data with logarithmical compression.

\section{Conclusions}

The use of 3-D ultrasound imaging modality in imageguided interventions is increasing. In many of these applications, it is important to determine the position of a needle and its tip. We propose a new technique for electrode localization in a 3-D ultrasound image. It permits one to automatically determine the electrode axis and the electrode tip. The axis is found by maximizing the PIP transform. A hierarchical mesh-grid search is used to accelerate this task. The tip position is determined by optimal thresholding of voxel intensities along the estimated axis.

The experiments performed on numerical phantoms show that the localization is robust with respect to the background noise and that accuracy does not depend significantly on the electrode orientation. Achieved localization accuracy is between 0.2 and $0.3 \mathrm{~mm}$ for real images, which is comparable to the axial resolution of the ultrasound system and similar to other state-of-the-art work. Our method is capable to localize very thin electrodes of diameter less than $1 \mathrm{~mm}$. The consistence of the results obtained on numerical phantoms and on real 3-D ultrasound data of a PVA phantom suggests that this approach is both robust and accurate under realistic conditions. We also have succeeded in localizing the needle in breast biopsy images. However, currently the region of interest must be specified very carefully.

The proposed method might be slow for some applications in its current implementation. The processing time of the current algorithm implementation in MATLAB is tens of minutes. However, it is easy to obtain substantial acceleration using a multiresolution approach [27]. Rewriting the method in a compiled language such as $\mathrm{C}$ or Java also will accelerate it substantially. 


\section{ACKNOWLEDGMENTS}

We would like to thank Daniel Buckton and Christian Perrey from GE Healthcare for providing the 3-D ultrasound data of breast biopsy.

\section{REFERENCES}

[1] A. Abati and A. Simsir, "Breast fine needle aspiration biopsy: Prevailing recommendations and contemporary practices," Clin. Lab. Med., vol. 25, no. 4, pp. 631-654, Dec. 2005.

[2] K. Kern, "Sentinel lymph node mapping in breast cancer using subareolarinjection of blue dye," J. Amer. Coll. Surg., vol. 189, no. 6 , pp. $539-545$, Dec. 1999.

[3] R. L. Alterman, D. Sterio, A. Beric, and P. J. Kelly, "Microelectrode recording during posteroventral pallidotomy: Impact on target selection and complications," Neurosurgery, vol. 44, no. 2, pp. 315-321, Feb. 1999.

[4] V. Horsley and R. H. Clarke, "The structure and functions of the cerebellum examined by a new method," Brain, vol. 31, pp. $45-124,1908$

[5] L. S. Scott, P. Douglas, G. H. Baltuch, and J. L. Jaggi, "Error analysis of MRI and Leksell stereotactic frame target localization in deep brain stimulation surgery," Stereotactic Functional Neurosurg., vol. 83, no. 1, pp. 1-5, 2005.

[6] A. Schweikard, G. Glosser, M. Bodduluri, M. J. Murphy, and J. R. Adler, "Robotic motion compensation for respiratory movement during radiosurgery," Comput. Aided Surg., vol. 5, no. 4, pp. $263-277,2000$.

[7] S. Krueger, F. Vogt, W. Hohenberger, D. Paulus, H. Niemann, and C. H. Schick, "Evaluation of computer-assisted image enhancement in minimal invasive endoscopic surgery," Methods Infor. Med., vol. 43, pp. 362-366, 2004.

[8] T. M. Peters, "Image-guidance for surgical procedures," Phys. Med. Biol., vol. 51, no. 14, pp. R505-R540, 2006.

[9] P. W. Glimcher, V. M. Ciaramitaro, M. L. Platt, H. M. Bayer, M. A. Brown, and A. Handel, "Application of neurosonography to experimental physiology," J. Neurosci. Methods, vol. 180, pp. 131-144, 2001.

[10] J. W. Cannon, J. A. Stoll, and I. S. Salgo, "Real time 3dimensional ultrasound for guiding surgical tasks," Comput. Aided Surg., vol. 8, pp. 82-90, 2003.

[11] K. J. Draper, C. C. Blake, D. B. Gowman, L. Downey, and A. Fenster, "An algorithm for automatic needle localization in ultrasound-guided breast biopsies," Med. Phys., vol. 27, pp. 1971-1979, 2000.

[12] P. M. Novotny, J. W. Cannon, and R. H. Howe, "Tool localization in 3-D ultrasound images," in Proc. Med. Image Comput. Comput.-Assisted Interven., vol. 2879, 2003, pp. 969-970.

[13] L. Tao, U. Castellani, A. Fusiello, and V. Murino, "3D acoustic image segmentation by a RANSAC-based approach," in Proc. Oceans Marine Technol. Ocean Sci. Conf., San Diego, Sep. 2003, pp. $1098-1101$.

[14] M. A. Fischler and R. C. Bolles, "Random sample consensus: A paradigm for model fitting with applications to image analysis and automated cartography," Commun. ACM, vol. 24, no. 6, pp. 381-395, June 1981.

[15] M. Barva, J. Kybic, J.-M. Mari, C. Cachard, and V. Hlaváč, "Automatic localization of curvilinear object in 3-D ultrasound images," in Proc. SPIE Int. Symp. Med. Imag., pp. 455-462, Feb. 2005

[16] M. Ding, H. N. Cardinal, W. Guan, and A. Fenster, "Automatic needle segmentation in 3-D ultrasound images," in Proc. SPIE, vol. 4681, May 2002, pp. 65-76.

[17] P. M. Novotny, J. A. Stoll, P. E. Dupont, and R. D. Howe, "Realtime visual servoing of a robot using three dimensional ultrasound," in Proc. IEEE Int. Conf. Robotics Automation (ICRA), 2007, pp. 2655-2660.

[18] P. M. Novotny, J. A. Stoll, N. V. Vasilyev, P. J. del Nido, P. E. Dupont, and R. D. Howe, "GPU based real-time instrument tracking with three dimensional ultrasound," Med. Image Analysis, vol. 11, no. 5, pp. 458-464, 2007.

[19] F. Natterer, The Mathematics of Computerized Tomography. Society for Industrial and Applied Mathematics, 2001.
[20] S. Helgason, The Radon Transform. 2nd ed. Boston: Birkhauser, 1999.

[21] R. Bulirsch and J. Stoer, Introduction to Numerical Analysis. New York: Springer-Verlag, 1991.

[22] E. Heitz, P. Perez, and P. Bouthemy, "Multiscale minimization of global energy functions in some visual recovery problems," Comput. Vision, Graphics, Image Process., vol. 59, no. 1, pp. 125-134, 1994.

[23] J. A. Jensen, "Field: A program for simulating ultrasound systems," in Medical and Biological Engineering and Computing. vol. 34, New York: Springer, 1996, pp. 351-353.

[24] J. A. Jensen and P. Munk, "Computer phantoms for simulating ultrasound B-mode and CFM images," Acoust. Imag., vol. 23, pp. 75-80, 1997.

[25] K. C. Chu and B. K. Rutt, "Polyvinyl alcohol cryogel: An idealphantom material for MR studies of arterial flow and elasticity," Magnet. Resonance Med., vol. 37, no. 2, pp. 314-319, 1997.

[26] J.-M. Mari and C. Cachard, "Acquire real-time RF digital ultrasound data from a commercial scanner," Electron. J. "Technical Acoustics", vol. 3, Jan. 2007.

[27] M. Uherčík, J. Kybic, C. Cachard, and H. Liebgott, "Multiresolution parallel integral transform for fast electrode localization in 3D ultrasound images," Research Report CTU-CMP-2007-24, Center for Machine Perception, K13133 FEE Czech Technical University, Prague, Czech Republic, Nov. 2007.

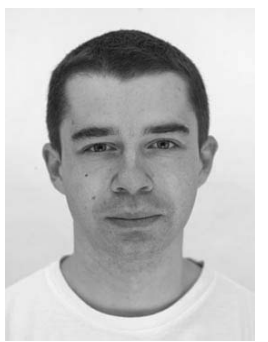

Martin Barva was born in 1977 in Czech Republic. He received an Ing. (M.Sc.) degree from the Faculty of Electrical Engineering and Communication of Brno University of Technology, Czech Republic, in 2002. In 2003, he obtained the DEA (M.Sc.) degree in signal and image processing from the INSA de Lyon, France. In 2007, he finished his Ph.D. studies under co-supervision at the research center CREATIS of the University Claude Bernard Lyon 1, France and in the Center for Machine Perception (CMP) of the Czech Technical University, Prague, Czech Republic.

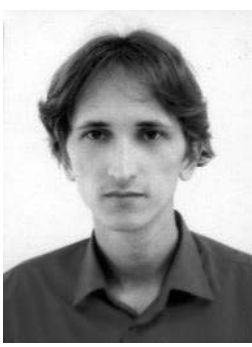

Marián Uherčík was born in 1981 in Slovakia. He received a Mgr. (M.Sc.) degree in informatics from the Faculty of Mathematics, Physics and Informatics of Comenius University in Bratislava in 2005. Currently he is doing Ph.D. work in the framework of Wide Area Research Training in Health Engineering (WARTHE) in the Center for Machine Perception (CMP) of the Faculty of Electrical Engineering of the Czech Technical University in Prague and in the research center CREATIS of the INSA de Lyon.

His research interests include medical applications and ultrasound imaging.

Jean-Martial Mari was born in 1976 in Saint Denis on Reunion Island (France). He received the Electrical Engineering Diploma of the Institut National des Sciences Appliquées (INSA) Lyon, France, in 2000 with a specialization in Signal Processing, and a Ph.D. degree in Acoustics at the University Lyon 1, France, in 2004. He joined the Medical Vision Laboratory, University of Oxford (UK), in 2005 working on ultrasound contrast agent and imaging artefacts correction, and the Bioengineering department at the Imperial College London (UK) in 2007 to study atherosclerosis. His general research interests include ultrasonic observation of surgical micro instruments, linear and non linear imaging, atherosclerosis prediction and detection, and technical acoustics. 


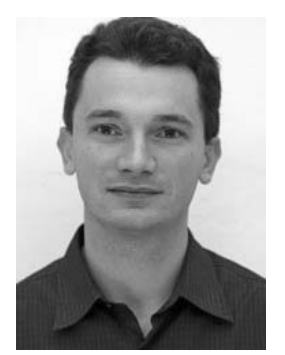

Jan Kybic (M'00) was born in Prague, Czech Republic, in 1974. He received the Mgr. (B.Sc.) and Ing. (M.Sc.) degrees with honors from the Czech Technical University, Prague, in 1996 and 1998, respectively. In 2001, he obtained the Ph.D. degree in biomedical image processing from Ecole Polytechnique Federale de Lausanne (EPFL), Switzerland, for his thesis on elastic image registration using parametric deformation models. Between October 2002 and February 2003, he held a postdoc research position in the Institut National de Recherche en Informatique et en Automatique (INRIA), SophiaAntipolis, France. Since 2003 he has been a research fellow with the Center for Machine Perception, Czech Technical University, Prague.

His research interests include signal and image processing, medical imaging, image registration, splines and wavelets, inverse problems, elastography, computer vision, numerical methods, algorithm theory, and control theory.

Jean-René Duhamel is director of the Cognitive Neuroscience Centre, a joint Centre de la Recherche Scientifique (CNRS)/University of Lyon Research Laboratory. He trained in psychology at McGill (B.A., 1980) and Concordia Universities (M.A., 1982) in Canada, and in Neuroscience at Aix-Marseille University, France, (Ph.D., 1987). After 4 years as a post-doctoral Fellow at the National Eye Institute, NIH Bethesda, Maryland, he joined the CNRS in 1991. He was based initially at the Collége de France in Paris, and moved to Lyon in 1998.

He now leads a multidisciplinary group of scientists using different experimental approaches (electrophysiology, neuroimaging, behavioral analysis, mathematical models) to study normal and pathological brain function.

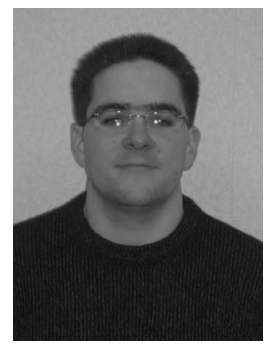

Hervé Liebgott received his Ph.D. degree in acoustics from the Institut National des Sciences Appliquées (INSA) Lyon in 2005. He also received an electrical ingenieur degree and masters in acoustics from the INSA de Lyon in 2002. He has worked at CREATIS since 2002, with the Ultrasonic Imaging team. He is currently associate professor at Université Claude Bernard Lyon where he teaches in the Electrical Department of the Technical Institute Université Lyon 1 Claude Bernard. He teaches informatics and mathematics in first and second year of electrical engineering.

His research deals with echography. More precisely he develops beam-forming techniques for ultrasound imaging in order to facilitate their processing. The processing methods that are investigated are motion estimation for tissue elasticity imaging by ultrasound (elastography), motion estimation for echocardiography, and detection of highly echogeneous objects in 3-D ultrasound data volumes.

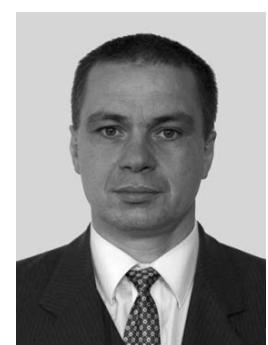

Václav Hlaváč was born in 1956 in Prague, Czech Republic. He is a professor at the Czech Technical University, Praha, Czech Republic, where he leads the Center for Machine Perception. He received an M.Sc. degree in 1981, and a Ph.D. degree in 1987 from the Czech Technical University, Prague.

His research interests are in 3-D computer vision and in both statistical and structural pattern recognition. He is a co-author of the books: Image Processing, Analysis, and Machine Vision, Thomson Learning, Toronto 2007; Ten Lectures on Statistical and Structural Pattern Recognition, Kluwer Academic Publishers, Dordrecht, The Netherlands, 2002.

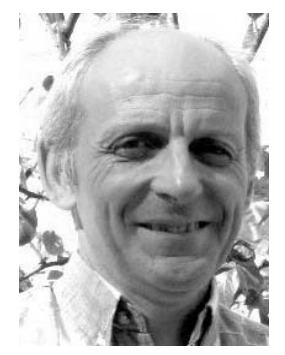

Christian Cachard received his Ph.D. degree in acoustics from Institut National des Sciences Appliquées (INSA), Lyon, in 1988, in the field of acoustic signal processing for underwater acoustics.

He is now a Professor at the Electrical Department of the Technical Institute Université Lyon 1 Claude Bernard. Since he joined the research laboratory CREATIS, his domain of interest includes ultrasound medical imaging, ultrasound contrast agent imaging, ultralocalization in 3-D ultrasound data. 